\title{
Eribulin Does Not Prevent Epithelial-to-Mesenchymal Transition in HT-29 Intestinal Epithelial Cells
}

\author{
Martin Leutenegger ${ }^{\mathrm{a}}$ Ramona Bruckner ${ }^{\mathrm{a}}$ Marianne R. Spalinger ${ }^{\mathrm{a}}$ Silvia Lang ${ }^{\mathrm{a}}$ \\ Gerhard Rogler ${ }^{a, b}$ Michael Scharl ${ }^{a, b}$ \\ ${ }^{a}$ Department of Gastroenterology and Hepatology, University Hospital Zurich, University of Zurich, \\ Zurich, Switzerland; ' Zurich Center for Integrative Human Physiology, University of Zurich, Zurich, Switzerland
}

\section{Keywords}

Crohn's disease · Fistulas · Eribulin ·

Epithelial-to-mesenchymal transition

\begin{abstract}
Background: Fistula formation affects up to 50\% of Crohn's disease (CD) patients and causes considerable morbidity. Current pharmacological management mainly includes antibiotics, immunosuppressives, and anti-TNF antibodies. CD fistulas develop from intestinal epithelial cells undergoing epithelial-to-mesenchymal transition (EMT). TGF $\beta$, the most important inducer of EMT, is detectable around CD fistula tracts and induces expression of the EMT-associated transcription factors SNAIL1 and SLUG as well as of IL-13. Conversely, together with TNF, IL-13 induces the expression of the transcription factor Ets-1 and $\beta 6$-integrin, which are associated with cell invasiveness. Eribulin is a synthetic derivative of halichondrin $B$, a large polyether macrolide, which reverses EMT in triple-negative breast cancer (TNBC) cells. Here, we investigated whether Eribulin might be a potential therapeutic option for CD fistulas via the inhibition of EMT. Summary: Chronic treatment with high concentrations of
\end{abstract}

\section{KARGER}

(c) 2018 S. Karger AG, Basel

E-Mail karger@karger.com

www.karger.com/iid
Eribulin ( $>5 \mathrm{ng} / \mathrm{ml}$ ) is toxic for intestinal epithelial cells (IEC), and Eribulin treatment does not decrease the mRNA expression of EMT markers in HT-29 monolayers nor prevent EMT in HT-29 spheroids. Together with TNF, Eribulin induces the expression of vimentin and SLUG mRNA in HT-29 spheroids but concomitantly also promotes E-cadherin expression. Key Messages: Our data suggest that the previously reported antimetastatic effect of Eribulin in TNBC by reversing EMT does not apply to IEC. Interestingly, Eribulin promotes E-cadherin expression, suggesting an additional mechanism. The increase of E-cadherin might point towards the described role for Eribulin in reversing EMT. Taken together, our data do not support a role for Eribulin as treatment option for CDassociated fistulas.

(c) 2018 S. Karger AG, Basel

\section{Introduction}

Fistulas represent a frequent and relapsing complication in Crohn's disease (CD) and can affect up to $50 \%$ of the patients at least once. Most common forms occur as perianal, enteroenteric, enterovesical, enterouterine, or 
enterovaginal and often cause considerable morbidity. Treatment options depend on symptom severity, fistula location, fistula complexity, and the presence of complications. The current pharmacological management includes mainly antibiotics, immunosuppressives, and anti-TNF antibodies. However, up to $30 \%$ of the perianal fistulas often require recurring surgical management [1], and therefore, the need for novel, nonsurgical treatment options is high.

Histomorphologically, CD fistulas consist of a central fissure, which is lined by granulation tissue [2]. About one-fourth of all CD fistulas have a central lining epithelium ("epithelialized fistulas"), which consists of flattened epithelial cells originating from the small intestine or colon without goblet cells, or of a narrow squamous epithelium present in enterocutaneous or perianal fistulas. In "nonepithelialized fistulas," some areas are lined with myofibroblast-like cells, the so-called transitional cells (TC), which transform into mucosal epithelial cells in the transitional zone. Functionally, the migratory potential of colonic lamina propria fibroblasts in CD patient intestine is reduced compared to non-IBD or ulcerative colitis patients resulting in a decreased wound healing potential [3]. Migration of intestinal epithelial cells (IEC), in turn, is induced [4].

To become mesenchymal-like myofibroblasts, IEC must undergo conversion in a process called epithelialto-mesenchymal transition (EMT). Here, the differentiated epithelial cells acquire a mesenchymal phenotype (e.g., enhanced migratory capacity, invasiveness, elevated resistance to apoptosis, and increased production of extracellular matrix, components) as demonstrated by the loss of epithelial markers, such as E-cadherin, and the expression of mesenchymal markers, such as vimentin [5]. EMT is also critically involved in the formation of TC [6]. Further, EMT has been demonstrated to play a key role for the development and invasiveness of epithelial carcinomas, such as breast cancer, lung cancer, or colorectal carcinoma [7-9]. We have recently demonstrated that EMT plays a critical role for fistula pathogenesis $[10,11]$. TGF $\beta$, which is the most important inducer of EMT that also induces the EMT-associated transcription factors SNAIL1 and SLUG [12], is expressed around CD fistula tracts. Additionally, TGF $\beta$ induces IL-13 expression via $\beta$-catenin and DKK-1 $[4,13]$. IL-13, together with TNF, then induces the expression of Ets-1 transcription factor and $\beta 6$-integrin, which are associated with cell invasiveness [14].

Eribulin is a synthetic derivative of halichondrin $\mathrm{B}$, a large polyether macrolide isolated from a rare marine
Japanese sponge. It inhibits cell proliferation by binding tubulin and destabilizing microtubule dynamics [15, 16]. Tubulin is sequestered into nonfunctional aggregates, leading to an irreversible arrest at cell cycle phase G2-M and after prolonged mitotic blockade finally to apoptosis [17]. In contrast to other antimicrotubule agents, Eribulin inhibits only microtubule growth without an effect on the shortening of microtubule in association with the sequestration of tubulin into aggregates [18]. Eribulin-induced apoptosis includes proteolytic activation of caspase- 3 and caspase- 9 and cleavage of the caspase-3-substrate poly(ADP-ribose) polymerase (PARP) [19].

To date, Eribulin is used as a treatment in triple-negative breast cancer (TNBC) [20]. The major part of TNBC have stem cell/basal-like properties [21]. Eribulin reverses EMT in TNBC cells. Amongst other genes, Eribulin treatment upregulates the expression of the epithelial marker E-cadherin and downregulates the expression of $\mathrm{N}$-cadherin, vimentin, and SLUG. Eribulin modulates the TGF $\beta$ signaling pathway via downregulation of Smad phosphorylation, which results in a reduced expression of EMT-related genes $[22,23]$. To assess a potentially therapeutic role for Eribulin in the treatment of CD-associated fistulas, we studied the effects of Eribulin on the human colon adenocarcinoma cells, HT-29, in different in vitro models.

\section{Material and Methods}

\section{Cell Culture}

Human HT-29 IEC were either grown as monolayers or spheroids in a humidified atmosphere with $10 \% \mathrm{CO}_{2}$ at $37^{\circ} \mathrm{C}$ in low glucose Dulbecco's modified Eagle medium (DMEM; Invitrogen, Carlsbad, CA, USA) containing 1,000 mg/L D-glucose, L-glutamine, and sodium pyruvate, as described below. The medium was additionally supplemented with $5 \%$ fetal calf serum (FCS; VWR, Darmstadt, Germany).

\section{Cell Viability Assay}

HT-29 cells were seeded onto 96-well plates. After $12 \mathrm{~h}$, the medium was replaced by low-glucose DMEM without additives. After $8 \mathrm{~h}$, the medium was replaced by low-glucose DMEM with $0.1,0.5,1,5,50,100$, or $500 \mathrm{ng} / \mathrm{mL}$ Eribulin as indicated and stimulated for $4,8,24,48$, and $72 \mathrm{~h}$. Cell supernatant was used to perform an LDH assay (Sigma-Aldrich, St. Louis, Missouri, USA), which quantifies LDH released after cell damage. Cell Counting Kit-8 (CCK8; Dojindo Molecular Technologies, Rockville, Maryland, USA) was used to evaluate cell proliferation and cytotoxicity. This assay quantifies the dehydrogenase activity in living cells, which is directly proportional to the number of living cells. 


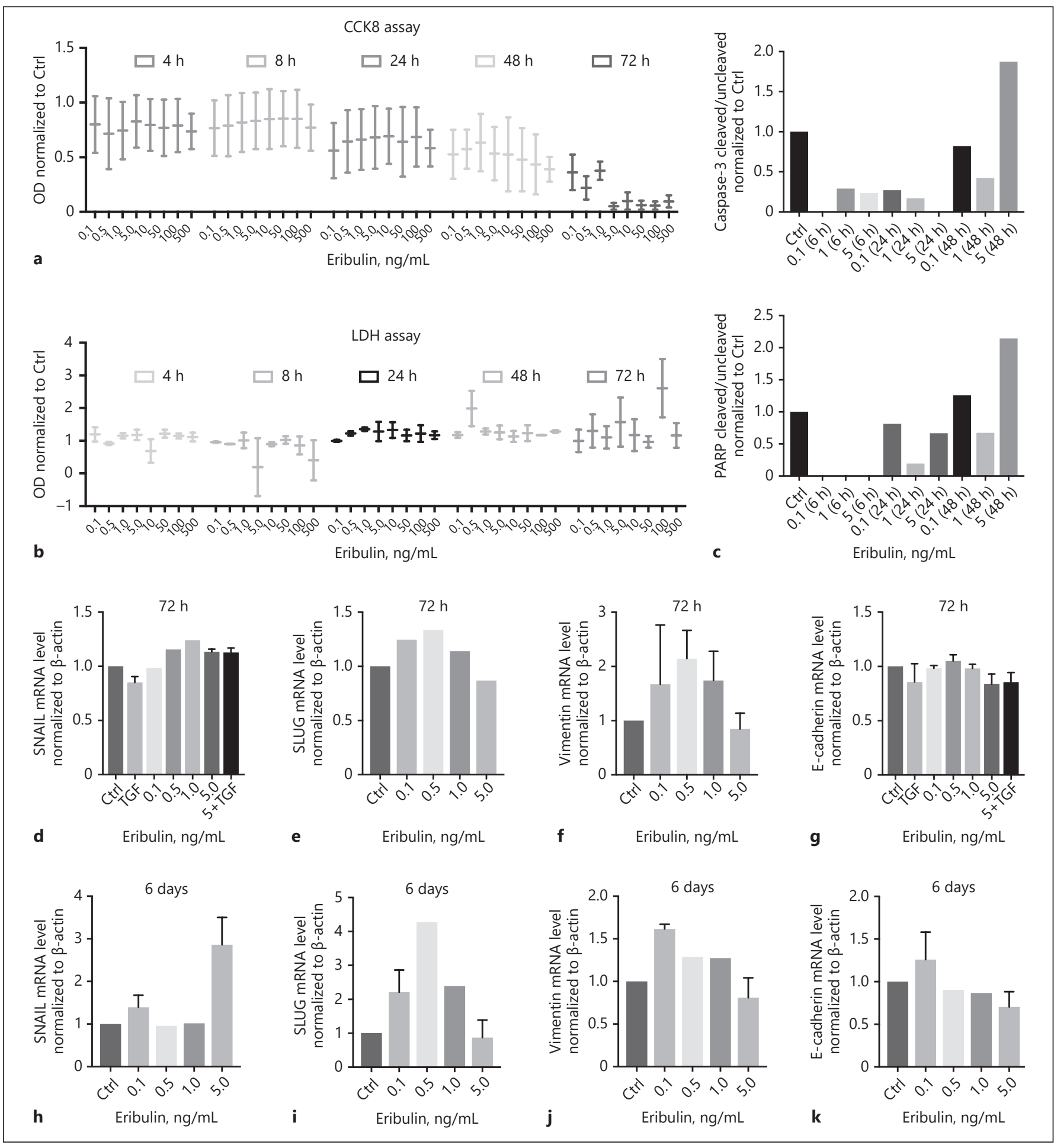

Fig. 1. Eribulin concentrations $>5 \mathrm{ng} / \mathrm{mL}$ and a stimulation time $>$ $48 \mathrm{~h}$ induce cell death in HT-29 IEC. Optical density (OD) values were normalized to control (Ctrl) using the Cell Counting Kit-8 (CCK8) assay (a, $n=2)$ and using the LDH assay $(\mathbf{b}, n=2)$. Ratio of cleaved/uncleaved caspase-3 and PARP after stimulation with Eribulin. Eribulin causes apoptosis in HT-29 IEC cells at higher concentrations and stimulation time points over $24 \mathrm{~h}$ (c). Numbers correspond to Eribulin concentrations $(\mathrm{ng} / \mathrm{mL})$ and the respective stimulation time. Relative mRNA expression of SNAIL, SLUG, vimentin, and E-cadherin after stimulation for $72 \mathrm{~h}(\mathbf{d}-\mathbf{g})$ and 6 days (h-k) with Eribulin. Numbers correspond to Eribulin concentrations (ng/mL). d $n=1-3$. e $n=1$. f $n=3$. $\mathbf{g} n=2-5$. h $n=1-3$. $\mathbf{i} n=1-3 . \mathbf{j} n=1-3 . \mathbf{k} n=1-3$. 
Stimulation of HT-29 Cells

After growing HT-29 cells in 12-well plates for $24 \mathrm{~h}$, the medium was removed and replaced by low-glucose DMEM without additives. HT-29 cells were stimulated with $50 \mathrm{ng} / \mathrm{mL}$ human recombinant TNFa (Calbiochem/Merck, Darmstadt, Germany; VWR) or with $50 \mathrm{ng} / \mathrm{mL}$ human recombinant TGF- $\beta 1$ (Calbiochem/Merck). After $24 \mathrm{~h}$, the medium was replaced with low-glucose DMEM containing 0.1, 0.5, 1, 5, 50, 100, or $500 \mathrm{ng} / \mathrm{mL}$ Eribulin for $72 \mathrm{~h}$ or 6 days, as indicated. TGF $\beta$ was only added to cells stimulated for $72 \mathrm{~h}$. Chemically synthesized Eribulin mesylate was obtained from Eisai Co., Ltd (Tsukuba, Japan).

\section{Spheroids as in vitro Model for EMT}

As an in vitro model for EMT, HT-29 cells were grown as spheroids [14]. $4.5 \times 10^{3}$ cells per spheroid were resuspended in 24 $\mu \mathrm{L}$ of HT-29 medium, seeded in Terasaki plates (Greiner-Microtest Plates, TC; EBIS/Huber \& Co) with 60 cavities and grown for 7 days. After 7 days, spheroids were stimulated with either $1 \mu \mathrm{L}$ of HT-29 medium without additives, $50 \mathrm{ng} / \mathrm{mL}$ of TNFa, as well as with $0.1,0.5,1,5,50$, and $200 \mathrm{ng} / \mathrm{mL}$ Eribulin. Spheroids were harvested after 7 days.

\section{Western Blotting}

An aliquot of each lysate was mixed with NuPAGE $4 \times$ LSD sample buffer (Invitrogen) and $50 \mathrm{~mm}$ dithiothreitol (Sigma) and boiled for $5 \mathrm{~min}$ at $98^{\circ} \mathrm{C}$. Proteins were separated by SDS-polyacrylamide gel electrophoresis and transferred onto nitrocellulose membranes (Invitrogen). Membranes were blocked with 3\% blocking solution and rabbit anti-PARP or rabbit anti-CASP3 antibody (1:1,000 in 3\% blocking solution; Abcam, Cambridge, UK) was applied overnight. The membranes were washed with Tris buffered saline containing 1\% Tween 20 (1\% TBST). HRP-labelled secondary anti-rabbit-IgG-antibody (1:4,000 in 3\% blocking solution; Santa Cruz Biotechnology, Dallas, TX, USA) was added for $90 \mathrm{~min}$, and the membranes were washed for $1 \mathrm{~h}$ with $1 \%$ TBST. Finally, immunoreactive proteins were detected using an enhanced chemoluminescence detection kit (GE Healthcare, Little Chalfont, UK).

\section{Real-Time Polymerase Chain Reaction}

Cell lysis was performed using RLT-buffer (Quiagen, Valencia, CA, USA) containing DTT. RNA was isolated using RNeasy Plus Mini Kit (Quiagen) according to the manufacturer's instructions. The RNA concentration was subsequently determined by absorbance at $230 \mathrm{~nm}$ using NanoDrop ND1000 (Thermo Scientific, Waltham, MA, USA). Complementary DNA synthesis was performed using a High-Capacity cDNA Reverse Transcription Kit

Fig. 2. After 7 days, Eribulin induces mRNA expression of vimentin and SNAIL but at the same time increases E-cadherin mRNA levels in HT-29 cells grown as spheroids. mRNA expression of SLUG (a), SNAIL (b), E-cadherin (c), and vimentin (d). Together, Eribulin and TNFa induce an even higher vimentin and SLUG mRNA expression, while at the same time they also increase Ecadherin expression levels and leave SNAIL expression unaltered in HT-29 spheroids after 7 days. mRNA expression of SLUG (e), SNAIL (f), E-cadherin $(\mathbf{g})$, and vimentin $(\mathbf{h})$. Eribulin reduces the
(Applied Biosystems, Foster City, CA, USA). TaqMan Assays and TaqMan Gene Expression Master Mix were obtained from Applied Biosystems. Real-time polymerase chain reaction was performed in a 7900HT Fast TR-PCR System using SDS 2.2 software (Applied Biosystems). Conditions were set on 45 cycles, denaturation of $1 \mathrm{~s}$ at $95^{\circ} \mathrm{C}$, and the annealing/extending at $60^{\circ} \mathrm{C}$ for $20 \mathrm{~s}$. Triplicate measurements were performed. Results were analyzed with the $\Delta \Delta$ CT method. The measured values were normalized to endogenously expressed $\beta$-actin.

\section{Statistical Analysis}

Data are shown as mean +/- S.E.M. for a series of $n$ experiments. Statistical analyses were performed (GraphPad Software, Inc., San Diego, CA, USA) by one-way analysis of variance followed by Dunnetts's multiple comparison post hoc test. $p<0.05$ was considered significant.

\section{Results}

\section{Eribulin Is Toxic for Intestinal Epithelial Cells}

Our first aim was to define non-cell-toxic concentrations of Eribulin, since the desired therapeutic effect in patients with CD fistulas would be to reverse or prevent the process of EMT in contrast to the desired cytotoxic effect in patients suffering from TNBC. First, we determined the effects of Eribulin on the viability of HT-29 IEC using a CCK-8 assay. We found that the treatment of HT-29 IEC for up to $24 \mathrm{~h}$ with Eribulin in a concentration range from $0.1-500 \mathrm{ng} / \mathrm{mL}$ did not affect cell viability. However, incubation times of 48 or $72 \mathrm{~h}$ at Eribulin concentrations of $5 \mathrm{ng} / \mathrm{mL}$ and higher reduced cell viability (Fig. 1a). An LDH release assay as a marker for cell death showed similar results (Fig. 1b). At Eribulin concentrations of $\geq 5 \mathrm{ng} / \mathrm{mL}$, the cells peeled off the wells, further confirming the toxic effect. This led us to use concentrations $<5 \mathrm{ng} / \mathrm{mL}$ for further experiments, since at lower concentrations, less Eribulin-induced cytotoxicity was detected.

We next studied molecular markers for apoptosis in HT-29 IEC. We detected increased cleavage of caspase-3

size of HT-29 spheroids at higher concentrations after 7 days of stimulation but does not induce EMT (i). TNFa treatment induces a fringed edge in the HT-29 spheroids, suggesting the onset of EMT. Co-treatment of Eribulin with TNFa reduces the size of the spheroids at increasing Eribulin concentrations (j). Numbers correspond to Eribulin concentrations $(\mathrm{ng} / \mathrm{mL})$. Three independent experiments were performed for all assays. Asterisks indicate significant differences versus the respective control. ${ }^{* *} p<0.01$, *** $p<0.001,{ }^{* * * *} p<0.0001$.

(For figure see next page.) 


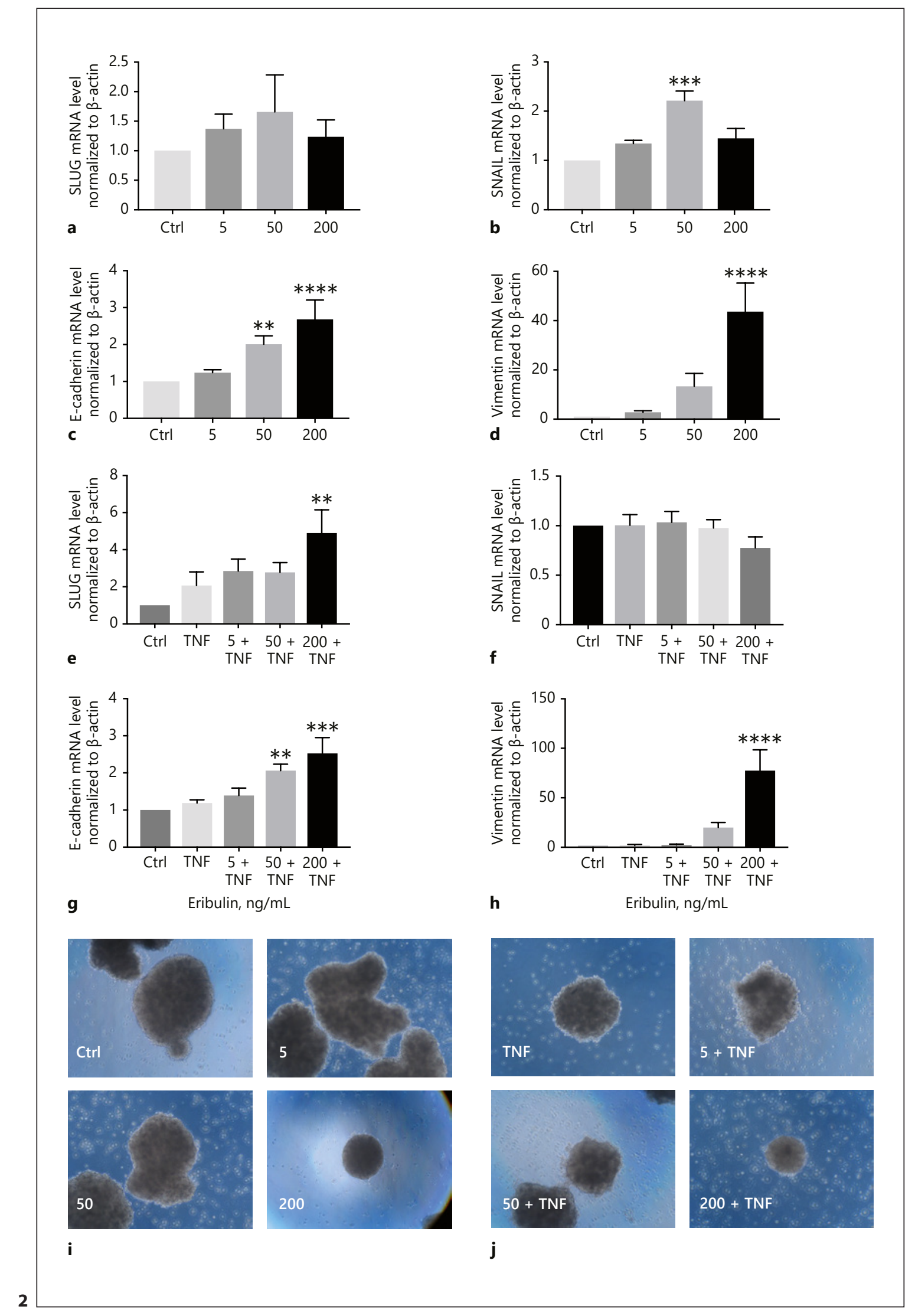


and PARP after treatment with Eribulin for $48 \mathrm{~h}$ at a concentration of $5 \mathrm{ng} / \mathrm{mL}$ (Fig. 1c). Lower concentrations of Eribulin did not promote cleavage of caspase- 3 or PARP in our cell model. Interestingly, Eribulin had no cell toxic effect upon shorter treatment intervals regardless of the concentration. We therefore concluded that Eribulin concentrations of max. $5 \mathrm{ng} / \mathrm{mL}$ were reasonable for further experiments.

\section{Eribulin Does Not Affect EMT Marker Expression in HT-29 Monolayers}

We then studied whether Eribulin would be able to affect mRNA expression levels of various EMT markers in HT-29 IEC monolayers. To address this aim, Eribulintreated HT-29 cells were analyzed for the mRNA expression levels of the EMT-associated transcription factors SNAIL and SLUG, of the epithelial marker E-cadherin, and of the mesenchymal marker vimentin.

After stimulation for $72 \mathrm{~h}$, treatment with Eribulin had no significant effect on E-cadherin mRNA expression levels, while SNAIL1, SLUG, and vimentin at least showed a minor trend for an increased expression (Fig. 1d-g). Of note, Eribulin did not affect TGF $\beta$-induced effects at $72 \mathrm{~h}$ treatment.

After stimulation of HT-29 IEC monolayers with Eribulin for 6 days, SNAIL, SLUG, and vimentin mRNA expression levels were increased in a dose-dependent manner, SLUG and vimentin mRNA expression levels were decreased at higher Eribulin concentrations. However, E-cadherin levels were not significantly altered but showed a trend towards reduced levels at higher Eribulin doses (Fig. 1h-k).

Previous studies showed that Eribulin reverses EMT in TNBC cells [22], while our results suggest that in IEC, Eribulin rather increased the expression of EMT-associated molecules and mesenchymal markers in HT-29 cell monolayers. $\mathrm{N}$-cadherin expression was also analyzed but was only expressed at very low levels, and no relative expression could be calculated.

\section{Eribulin Does Not Prevent EMT in HT-29 Spheroids}

We next studied the effects of Eribulin in our well-established EMT cell model using HT-29 spheroids. In contrast to monolayers, spheroids allow to study the effect on tight-junction integrity and cell proliferation in a more physiological manner. HT-29 cells were seeded as spheroids and treated after 7 days with Eribulin for additional 7 days. Eribulin did not significantly alter the expression levels of mRNA for the EMT marker gene SLUG and $\beta 6$ integrin. At higher concentrations, Eribulin treatment even enhanced mRNA expression of the mesenchymal markers vimentin, SLUG, and SNAIL but also of the epithelial marker E-cadherin. None of the mRNA expression for the EMT markers was reduced (Fig. 2a-d). Microscopically, the spheroids appeared smaller, but confined by a thin border, with increasing concentrations of Eribulin (Fig. 2i).

We then induced EMT in our HT-29 spheroids by stimulating with TNFa at a concentration of $50 \mathrm{ng} / \mathrm{mL}$ for 7 days, since it has been shown that TNFa induces the expression of factors associated with EMT [14]. HT-29 cells were seeded as spheroids and stimulated with Eribulin and TNFa. TNFa-treatment induced the mRNA expression of the EMT marker SLUG and vimentin, clearly indicating the onset of EMT after 7 days. However, TNFainduced effects were not diminished by co-treatment with Eribulin, and high doses of Eribulin even enhanced TNFa-induced expression of SLUG and vimentin, while leaving SNAIL expression unaltered. (Fig. 2e-h). Interestingly, E-cadherin mRNA expression was also increased after Eribulin co-treatment, while it was not influenced by TNF $\alpha$-treatment alone (Fig. $2 \mathrm{~g}$ ). $\beta 6$-integrin mRNA levels were unaltered by Eribulin and TNFa-treatment either alone or in combination (data not shown). Microscopically, spheroids revealed a rather fringed boarder after stimulation with TNFa, suggesting the onset of EMT (Fig. 2j). Eribulin and TNFa cotreatment reduced the size of the spheroids with increasing Eribulin concentrations. $\mathrm{N}$-cadherin expression was also analyzed but was only expressed at very low levels, and no relative expression could be calculated.

\section{Discussion}

EMT has been shown to play a pivotal role for the development of $\mathrm{CD}$-associated intestinal fistulas. Eribulin is used in the treatment of TNBC and reverses the onset of EMT. Here, we investigated whether Eribulin might be able to affect EMT in IEC, which might provide the rationale for the use of Eribulin in the treatment of CD-associated fistulas.

In the present study, we have demonstrated that Eribulin has cytotoxic effects on HT-29 cells in high doses and upon prolonged treatment duration, but in low concentrations up to $5 \mathrm{ng} / \mathrm{mL}$, Eribulin is not cytotoxic for IEC and might be suitable for targeting EMT in IEC. However, when grown as monolayers, Eribulin was not able to reverse induced EMT in HT-29 cells. This is contrary to previous studies, showing that Eribulin reverses 
EMT in TNBC cells [22]. In our hands, Eribulin even increases the expression of EMT markers in IEC. Interestingly, in our HT-29 spheroid model, a well-established cell model for EMT, we found that Eribulin and TNFa have synergistic effects on the expression of mesenchymal markers or EMT-associated genes. A plausible explanation might be the loosening of cell-cell contacts because of EMT and subsequently an enlarged contact surface with Eribulin. Higher concentrations of Eribulin were needed to see effects on gene regulation in spheroids, as compared to HT-29 monolayers, probably because of tighter cell-cell contacts and a more epithelial-like phenotype of the spheroids. In conclusion, our data have shown that Eribulin does not reverse EMT in HT-29 IEC.

In summary, the data from this study suggest that the previously reported antimetastatic effect of Eribulin in TNBC, by reversing EMT, does most likely not apply to intestinal epithelial cells. A reason for this result might be that HT-29 IEC already have a distinct epithelial phenotype more similar to a physiologically configured epithelium, while TNBC seems to have a more stem-cell like mesenchymal behavior corresponding to the cells of an aggressively metastatic type of cancer. It has already been shown that the expression of some EMT marker genes predicts Eribulin sensitivity in some cell lines [24]. Interestingly, Eribulin not only enhanced the mRNA expression of the EMT markers SLUG and vimentin but also promoted E-cadherin expression, a marker associated with an epithelial phenotype. This suggests that in IEC, Eribulin also induces other mechanisms than the already described ones involving EMT. Eribulin affects gene expression in different ways, holding a balance. On the one hand, there is the specific effect on microtubule structure and therefore internal cell transport mechanisms probably altering E-cadherin expression. On the other hand, there is the cytotoxic and proapoptotic effect causing inflammatory responses and probably altering SLUG expression. The increase of E-cadherin might point towards the also described role for Eribulin in reversing EMT. The low $\mathrm{N}$-cadherin mRNA expressions could also be partially explained by the distinct epithelial phenotype of the HT-29 cells. All in all, our data suggest that Eribulin is likely not an appropriate therapeutic option for the treatment of CD-associated fistulas.

\section{Acknowledgements}

This research was supported by research grants from the Swiss National Science Foundation to M.S. (grant No. 314730-146204, grant No. 314730_166381, and grant No. CRSII3_154488/1) and the Swiss IBD Cohort (grant No. 3347CO-108792) to G.R., a grant from the European Crohn's and Colitis Foundation (ECCO) to M.R.S., and a grant from the International Organisation for Research in IBD (IOIBD) to M.S. The funding institutions had no role in study design and data interpretation.

\section{Statement of Ethics}

No ethical approval was needed to conduct the study.

\section{Disclosure Statement}

The authors declare no financial conflicts of interests.

\section{References}

1 Nielsen OH, Rogler G, Hahnloser D, Thomsen OO: Diagnosis and management of fistulizing Crohn's disease. Nat Clin Pract Gastroenterol Hepatol 2009;6:92-106.

2 Bataille F, Klebl F, Rümmele P, Schroeder J, Farkas S, Wild PJ, et al: Morphological characterisation of Crohn's disease fistulae. Gut 2004;53:1314-1321.

3 Meier JK, Scharl M, Miller SN, Brenmoehl J, Hausmann M, Kellermeier S, et al: Specific differences in migratory function of myofibroblasts isolated from Crohn's disease fistulae and strictures. Inflamm Bowel Dis 2011; 17:202-212.

4 Scharl M, Rogler G: Pathophysiology of fistula formation in Crohn's disease. World J Gastrointest Pathophysiol 2014;5:205-212.
5 Kalluri R, Weinberg RA: The basics of epithelial-mesenchymal transition. J Clin Invest 2009;119:1420-1428.

6 Bataille F, Rohrmeier C, Bates R, Weber A, Rieder F, Brenmoehl J, et al: Evidence for a role of epithelial mesenchymal transition during pathogenesis of fistulae in Crohn's disease. Inflamm Bowel Dis 2008;14:1514-1527.

7 Bates RC: Colorectal cancer progression: integrin alphavbeta 6 and the epithelial-mesenchymal transition (EMT). Cell Cycle 2005;4: 1350-1352.

8 Scharl M, Frei P, Frei SM, Biedermann L, Weber A, Rogler G: Epithelial-to-mesenchymal transition in a fistula-associated anal adenocarcinoma in a patient with long-standing Crohn's disease. Eur J Gastroenterol Hepatol 2014;26:114-118.
9 Arias AM: Epithelial mesenchymal interactions in cancer and development. Cell 2001; 105:425-431.

10 Scharl M, Weber A, Fürst A, Farkas S, Jehle E, Pesch T, et al: Potential role for SNAIL family transcription factors in the etiology of Crohn's disease-associated fistulae. Inflamm Bowel Dis 2011;17:1907-1916.

11 Scharl M, Frei S, Pesch T, Kellermeier S, Arikkat J, Frei $\mathrm{P}$, et al: Interleukin-13 and transforming growth factor $\beta$ synergise in the pathogenesis of human intestinal fistulae. Gut 2013;62:63-72

12 Zavadil J, Böttinger EP: TGF-beta and epithelial-to-mesenchymal transitions. Oncogene 2005;24:5764-5774. 
13 Frei SM, Hemsley C, Pesch T, Lang S, Weber A, Jehle E, et al: The role for dickkopf-homo$\log -1$ in the pathogenesis of Crohn's diseaseassociated fistulae. PloS One 2013;8:e78882.

14 Frei SM, Pesch T, Lang S, Weber A, Jehle E, Vavricka SR, et al: A role for tumor necrosis factor and bacterial antigens in the pathogenesis of Crohn's disease-associated fistulae. Inflamm Bowel Dis 2013;19:2878-2887.

15 Bai RL, Paull KD, Herald CL, Malspeis L, Pettit GR, Hamel E: Halichondrin B and homohalichondrin B, marine natural products binding in the vinca domain of tubulin. Discovery of tubulin-based mechanism of action by analysis of differential cytotoxicity data. J Biol Chem 1991;266:15882-15889.

16 Okouneva T, Azarenko O, Wilson L, Littlefield BA, Jordan MA: Inhibition of centromere dynamics by eribulin (E7389) during mitotic metaphase. Mol Cancer Ther 2008;7: 2003-2011.
17 Kuznetsov G, Towle MJ, Cheng H, Kawamura $\mathrm{T}$, TenDyke $\mathrm{K}$, Liu $\mathrm{D}$, et al: Induction of morphological and biochemical apoptosis following prolonged mitotic blockage by halichondrin B macrocyclic ketone analog E7389. Cancer Res 2004;64:5760-5766.

18 Jordan MA, Kamath K, Manna T, Okouneva T, Miller HP, Davis C, et al: The primary antimitotic mechanism of action of the synthetic halichondrin E7389 is suppression of microtubule growth. Mol Cancer Ther 2005;4: 1086-1095.

19 Jain S, Cigler T: Eribulin mesylate in the treatment of metastatic breast cancer. Biologics 2012;6:21-29.

20 Cortes J, O'Shaughnessy J, Loesch D, Blum JL, Vahdat LT, Petrakova K, et al: Eribulin monotherapy versus treatment of physician's choice in patients with metastatic breast cancer (EMBRACE): a phase 3 open-label randomised study. Lancet 2011;377:914-923.
21 Perou CM, Sørlie T, Eisen MB, van de Rijn M, Jeffrey SS, Rees CA, et al: Molecular portraits of human breast tumours. Nature 2000;406: 747-752.

22 Yoshida T, Ozawa Y, Kimura T, Sato Y, Kuznetsov G, Xu S, et al: Eribulin mesilate suppresses experimental metastasis of breast cancer cells by reversing phenotype from epithelial-mesenchymal transition (EMT) to mesenchymal-epithelial transition (MET) states. Br J Cancer 2014;110:1497-1505.

23 Takano S, Kanai F, Jazag A, Ijichi H, Yao J, Ogawa $\mathrm{H}$, et al: Smad4 is essential for downregulation of E-cadherin induced by TGF-beta in pancreatic cancer cell line PANC-1. J Biochem 2007;141:345-351.

24 Dezső Z, Oestreicher J, Weaver A, Santiago S, Agoulnik S, Chow J, et al: Gene expression profiling reveals epithelial mesenchymal transition (EMT) genes can selectively differentiate eribulin sensitive breast cancer cells. PloS One 2014;9:e106131. 